\title{
Total Nicotine Equivalent
}

National Cancer Institute

\section{Source}

National Cancer Institute. Total Nicotine Equivalent. NCI Thesaurus. Code C116393.

The sum of the molecules of nicotine and each of its metabolites, including cotinine, trans 3'-hydroxycotinine and their respective glucuronide conjug ates, detected in a biological sample. 